\title{
Three problems for polynomials of small measure
}

\author{
by \\ Artūras Dubickas (Vilnius)
}

1. Introduction. Let $f(x)=\sum_{k=0}^{D} a_{k} x^{k}$ be a nonzero polynomial with complex coefficients. To measure the complexity ("height") of the polynomial $f$ one may use the notions of the length of $f, L(f)=\sum_{k=0}^{D}\left|a_{k}\right|$, the "classical" height of $f, H(f)=\max _{0 \leq k \leq D}\left|a_{k}\right|$, the maximum of the modulus of $f$ in the unit circle, $\|f\|=\max _{|z| \leq 1}|f(z)|$, the Mahler measure of $f$,

$$
M(f)=\exp \left(\int_{0}^{2 \pi} \log \left|f\left(e^{i t}\right)\right| d t\right),
$$

or say the value of $f$ at unity, $f(1)$.

Set $r_{\varrho}(f)$ for the order of vanishing of $f$ at a complex number $\varrho$. Below, we shall use this notation for only two values of $\varrho: \varrho=1$ and $\varrho=-1$.

A nonzero algebraic number $\alpha \in \overline{\mathbb{Q}}^{*}$ is described by its unique normalised minimal polynomial $P=P_{\alpha}$, whose coefficients are relatively prime integers and whose leading coefficient is positive. The minimal polynomial $P$ is irreducible over the field of rational numbers $\mathbb{Q}$. Let $\mathcal{N}: \overline{\mathbb{Q}}^{*} \rightarrow \mathbb{Q}$ be the norm function. For every $\alpha \in \overline{\mathbb{Q}}^{*}$, we have

$$
|\mathcal{N}(\alpha-1)| \leq|P(1)| \leq\|P\| \leq L(P),
$$

where $P=P_{\alpha}$.

We can now state three related problems for polynomials of small Mahler measure.

Multiplicity Problem. For a given set of complex numbers $\mathcal{A}$, find or estimate

$$
r_{\varrho}(\mathcal{A}, D)=\max r_{\varrho}(f),
$$

where the maximum is taken over all nonzero polynomials $f$ of degree $D$ with coefficients in the set $\mathcal{A}$.

2000 Mathematics Subject Classification: 11R04, 11R09, 11B83, $12 \mathrm{D} 05$. 
Really general sets $\mathcal{A}$ do only occur in the second statement of Theorem 3 . In most of the cases we deal with the set

$$
\mathcal{H}=\{-H,-H+1, \ldots, H-1, H\},
$$

where $H$ is a positive integer, and its subsets $\mathcal{E}=\{-1,0,1\}, \mathcal{B}=\{0,1\}$, and $\mathcal{F}=\{-1,1\}$.

Height PRoblem. Estimate the maximum of some "height" (say $|P(1)|$, $H(P)$, or $L(P)$ ) over all irreducible integer polynomials $P$ of degree $d$ and of Mahler measure at most $M$.

Approximation problem. Estimate the minimum of $|\alpha+1|$ over all algebraic numbers of degree $d$ and of Mahler measure at most $M$.

In the subsequent sections we discuss the above problems and give some new results. Some of these (see Sections 3 and 4) show that the problems are indeed related, and the progress in each of the three would sharpen either upper or lower bounds in the remaining two.

2. Multiplicity problem. The multiplicity problem for integer polynomials of bounded height, that is, with coefficients in the set $\mathcal{H}$ is an old one. Note that $r_{1}(\mathcal{H}, D)=r_{-1}(\mathcal{H}, D)$, since $\mathcal{H}=-\mathcal{H}$. Various bounds for $r_{1}(\mathcal{H}, D)$ were obtained by Bloch and Pólya [7], Schur [28], Mignotte [23], Bombieri and Vaaler [8], [9], Amoroso [2], Borwein, Erdélyi and Kós [10]. In particular, if $\varepsilon>0$ for the $\{-1,0,1\}$ polynomials we have the bounds

$$
(2-\varepsilon) \sqrt{\frac{D \log 2}{\log D}}<r_{1}(\mathcal{E}, D)<\frac{21}{13} \sqrt{D}
$$

for all $D$ sufficiently large. Here the lower bound follows from the work of Bombieri and Vaaler [8] (see also [18] for a different proof). The upper bound (see [20]) strengthens the constant 16/7 obtained by Borwein, Erdélyi and Kós [10].

The problem of closing the remaining sublogarithmic gap is almost certainly a difficult one. In particular, the sharpening of the lower bound would have considerable applications in Diophantine approximation. Borwein and Mossinghoff [11] gave some computational results for the degrees of polynomials with coefficients in $\{-1,0,1\}$ and prescribed vanishing at 1 .

In some cases the magnitude of $r_{1}(\mathcal{A}, D)$ is considerably smaller. For polynomials with $\{-1,1\}$ coefficients, Boyd [13] proved the inequalities

$$
\log D \ll r_{1}(\mathcal{F}, D)<(1+\varepsilon) \frac{(\log D)^{2}}{\log \log D},
$$

where $\varepsilon>0$, and $D$ is a sufficiently large integer. His upper bound is true for every set $\mathcal{F}$ of odd integers which is bounded by an absolute constant. 
Clearly, $r_{1}(\mathcal{B}, D)=0$, because the polynomials with $\{0,1\}$ coefficients do not vanish at 1 . For $r_{-1}(\mathcal{B}, D)$ we can give an almost complete answer. Below [...] stands for the integral part.

Theorem 1. Let $D \geq 2$ be a positive integer. Then either

$$
r_{-1}(\mathcal{B}, D)=\left[\frac{\log D}{\log 2}\right], \quad \text { or } \quad r_{-1}(\mathcal{B}, D)=\left[\frac{\log D}{\log 2}\right]-1 .
$$

Furthermore, each possibility occurs infinitely often.

Proof. The upper bound

$$
r_{-1}(\mathcal{B}, D) \leq \frac{\log (D+1)}{\log 2}
$$

is given in [10]. We shall replace $D+1$ by $D$.

It follows easily that $r_{-1}(\mathcal{B}, 1)=r_{-1}(\mathcal{B}, 2)=1$. Suppose that $2^{r}>$ $D>2$, where $r=r_{-1}(\mathcal{B}, D)$. Let $g$ be a nonzero polynomial with $\{0,1\}$ coefficients such that $r_{-1}(g)=r$. Clearly,

$$
g(x) \neq 1+x+x^{2}+\ldots+x^{D},
$$

since $r>1$. At least one coefficient of $g$, therefore, is equal to 0 . Hence

$$
2^{r} \leq g(1) \leq D
$$

a contradiction. Since $r$ is an integer, we deduce the inequality

$$
r=r_{-1}(\mathcal{B}, D) \leq\left[\frac{\log D}{\log 2}\right] .
$$

Set $b_{k}=\left(2^{k+1}+(-1)^{k}\right) / 3$. Of course, $\left\{b_{k}\right\}_{k=1,2, \ldots}$ is a sequence of positive odd integers. For any positive integer $k$ one can easily verify the inequality

$$
b_{1}+\ldots+b_{k}<b_{k+1}
$$

(in fact, the sum on the left hand side is $b_{k+1}-1$ or $b_{k+1}-2$ according as $k$ is even or odd). Therefore,

$$
B_{r}(x)=\prod_{k=1}^{r}\left(x^{b_{k}}+1\right)
$$

is a polynomial with coefficients in the set $\mathcal{B}=\{0,1\}$. Set now

and

$$
r=\left[\frac{\log (3 D / 4)}{\log 2}\right]
$$

$$
B_{r}^{*}(x)=x^{D-b_{1}-b_{2}-\ldots-b_{r}} \prod_{k=1}^{r}\left(x^{b_{k}}+1\right) .
$$

Since

$$
b_{1}+\ldots+b_{r} \leq b_{r+1}-1<\frac{4}{3} \cdot 2^{r} \leq D,
$$

$B_{r}^{*}(x)$ is a $\{0,1\}$ polynomial of degree $D$. 
Note that

$$
r_{-1}\left(B_{r}^{*}\right)=r=\left[\frac{\log (3 D / 4)}{\log 2}\right]=\left[\frac{\log D}{\log 2}-0.415 \ldots\right] \geq\left[\frac{\log D}{\log 2}\right]-1 .
$$

This shows that there are only two possibilities for $r_{-1}(\mathcal{B}, D)$. Furthermore, if

$$
\left\{\frac{\log D}{\log 2}\right\} \geq 2-\frac{\log 3}{\log 2}=0.415 \ldots,
$$

where $\{\ldots\}$ stands for the fractional part, then we have the first possibility, namely,

$$
r_{-1}(\mathcal{B}, D)=r=\left[\frac{\log (3 D / 4)}{\log 2}\right]=\left[\frac{\log D}{\log 2}\right] .
$$

Clearly, there are an infinite number of such integers $D$.

Suppose now that $D$ is a power of $2: D=2^{m}, D \geq 8$. We claim that

$$
r_{-1}\left(\mathcal{B}, 2^{m}\right)=m-1 \text {. }
$$

Indeed, assume that there is a $\{0,1\}$ polynomial $g$ of degree $D=2^{m}$ for which

$$
r_{-1}(g)=m \text {. }
$$

Then there is a coefficient of $g$ equal to zero, for otherwise $g(-1) \neq 0$. Suppose that there is only one such coefficient. Since $m \geq 3$, we see that $g(-1)=0, g^{\prime}(-1)=0$ and $g^{\prime \prime}(-1)=0$. From the first two of these equalities one can easily find that

$$
g(x)=\frac{x^{D+1}-1}{x-1}-x^{D / 2} .
$$

But then

$$
g^{\prime \prime}(-1)=\frac{D^{2}+2 D}{4} \neq 0,
$$

a contradiction. Thus, among the coefficients of $g$ there are at least two zeros. Then, as above,

$$
2^{m}=2^{r_{-1}(g)} \leq D-1<2^{m},
$$

a contradiction. Consequently, for every $m \geq 3$ we have the second possibility, namely, $r_{-1}\left(\mathcal{B}, 2^{m}\right)=m-1$.

Note that Theorem 1 is true for every set $\{0, b\}$, where $b$ is a nonzero complex number, by considering the $\{0,1\}$ polynomials $g / b$ instead of the $\{0, b\}$ polynomials $g$.

3. Height problem. For the height and approximation problems we assume that $1<M<\exp \left(d^{1-\delta}\right)$, where $\delta$ is a small positive constant. Here 
we use the letter $d$ instead of $D$ for the degree to show that the polynomials involved are irreducible.

Mignotte [24] showed that the inequality

$$
L(P) \leq(2 d+1) M \exp \left(\sqrt{2 d(4+\log d) \log \left((2 d+1)^{1 / 2} M\right)}\right)
$$

holds for irreducible integer polynomials $P$ of degree $d$ and of measure at most $M$. For large $M$, say $M \geq e^{d} / \sqrt{d}$, the Liouville inequality $L(P) \leq$ $2^{d} M(P)$ gives a stronger bound. This is also the case for cyclotomic polynomials $(M=1)$. Amoroso [5] and the author [21] slightly improved the constants in Mignotte's inequality. For instance, in the range $1<M<d^{\theta}$ the inequality

$$
H(P) \leq\|P\| \leq L(P)<\exp ((1+\theta) \sqrt{d / 2} \log d)
$$

is true for all $d$ sufficiently large [21].

For the height $P(1)$, Bugeaud [14] obtained the bound

$$
|P(1)| \leq M^{4} \exp (2 \sqrt{d \log d \log M}) .
$$

The proof is based on the analytical inequality

$$
\left|\prod_{k=1}^{K-1}\left(z^{k}-1\right)^{K-k}\right| \leq \max \{1,|z|\}^{K\left(K^{2}-1\right) / 6} K^{K / 2},
$$

where $z$ is a complex number and $K \geq 2$ is an integer. The above inequality follows from Hadamard's inequality, by representing the polynomial on the left hand side as

$$
z^{-K(K-1)(K-2) / 6} \operatorname{Det}\left(x^{i j}\right)_{0 \leq i, j \leq K-1} .
$$

In some sense this proof can be compared with Zagier's method [32] used for a lower bound of $(M(\alpha) M(1-\alpha))^{1 / d}$.

We remark that there is more information in the above inequality than just an upper bound for $|P(1)|$. Taking the product over all $d$ conjugates of $\alpha \in \overline{\mathbb{Q}}^{*}, \alpha \neq 1$, with leading coefficient $a$ one easily gets the inequality

$$
\sum_{k=1}^{K-1}\left(1-\frac{k}{K}\right) \log \left|\mathcal{N}\left(\alpha^{k}-1\right)\right| \leq \frac{K^{2}-1}{6} \log \left(\frac{M(\alpha)}{a}\right)+\frac{d}{2} \log K .
$$

This "one-line proof" sharpens the essentially Blanksby and Montgomery inequality [6] (see Silverman [29], [30], [31]) obtained by using the Fourier averaging techniques. Silverman [30] speculates that such an inequality can be used to approach the Lehmer conjecture.

The author used some more subtle determinants [16], [17] which gave the inequality

$$
|P(1)|<\exp \left(\left(\frac{\pi}{4}+\varepsilon\right) \sqrt{d \log d \log M}\right)
$$


for any $\varepsilon>0$ and for all $d$ sufficiently large. These proved to be useful in estimating the number of cyclotomic factors of a polynomial [19]. The latter problem was first investigated by Schinzel [27]. See also the paper of Pinner and Vaaler [26].

Amoroso [1] showed that these bounds are not far from being sharp. He proved that for $\varepsilon>0$ the inequality

$$
|P(1)|>\exp ((1-\varepsilon) \sqrt{d \log M(P)})
$$

has infinitely many solutions in irreducible integer polynomials $P$ such that $d^{2} / 4 \leq M(P) \leq d^{2} / 2$. By simplifying his example, we show that it also has infinitely many solutions for smaller $M(P)$, say $d<M(P)<(1+\varepsilon) d$. It would be of interest to reduce the upper bound for $M(P)$ to, for instance, $M(P) \leq \sqrt{d}$, if possible.

For every integer $q \geq 2$, set

$$
d=\sum_{p \leq q}(p-1)
$$

where the sum is taken over primes. Let $p(d)$ be a prime number greater than $d$, and let

$$
Q_{d}(x)=x^{1+d-p(d)} \frac{d}{d x}\left(x^{p(d)-d} \prod_{p \leq q}\left(1+x+x^{2}+\ldots+x^{p-1}\right)\right) .
$$

Clearly, $Q_{d}(x)$ is an integer polynomial of degree $d$.

THEOREM 2. $Q_{d}$ is an irreducible integer polynomial such that $M\left(Q_{d}\right)=$ $p(d)$. Furthermore, for any $\varepsilon>0$ and $d$ sufficiently large we have

$$
Q_{d}(1)>\exp ((1-\varepsilon) \sqrt{d \log d}) .
$$

Note that if $p(d)<\exp (\varepsilon \sqrt{d \log d})$, then for any root $\alpha$ of $Q_{d}$,

$$
\mathcal{N}(\alpha-1)=\frac{Q_{d}(1)}{p(d)}>\exp ((1-2 \varepsilon) \sqrt{d \log d}),
$$

because the coefficients of $Q_{d}$ are all nonnegative. Also, if say $p(d) \leq d^{1+\varepsilon}$, then

$$
\mathcal{N}(\alpha-1)>\exp ((1-2 \varepsilon) \sqrt{d \log M(\alpha)}) .
$$

Proof of Theorem 2. The polynomial

$$
x^{p(d)-d} \prod_{p \leq q}\left(1+x+\ldots+x^{p-1}\right)
$$

has no multiple roots on the unit circle $|z|=1$. Hence, the zeros of $Q_{d}$ are all strictly inside the unit circle, since the smallest closed convex set containing all the zeros of a polynomial also contains all the zeros of the derivative of 
the polynomial. Suppose that

$$
Q_{d}(x)=p(d) x^{d}+\ldots+p(d)-d
$$

is reducible. From the fact that $p(d)$ is a prime number, we deduce that there is an irreducible integer polynomial $Q^{*}$ with the leading coefficient $p(d)$ so that $Q^{*} \mid Q_{d}$. The remaining zeros of the ratio $Q_{d} / Q^{*}$ must contain a full set of conjugates of an algebraic integer. This is, however, impossible, because they are all strictly inside the unit circle $|z|<1$, and $Q_{d}(0) \neq 0$. Consequently, $\operatorname{deg} Q_{d}=d$ and $M\left(Q_{d}\right)=p(d)$.

For the last inequality, note that if $\varepsilon>0$ and if $q$ is sufficiently large then

$$
d=\sum_{p \leq q}(p-1)<\left(1+\frac{\varepsilon}{2}\right) \frac{q^{2}}{2 \log q} .
$$

Hence $q>(1-\varepsilon / 2) \sqrt{d \log d}$. Since the coefficients of $Q_{d}$ are all nonnegative, it follows that

$$
Q_{d}(1)>\prod_{p \leq q} p>\exp \left(\left(1-\frac{\varepsilon}{2}\right) q\right)>\exp ((1-\varepsilon) \sqrt{d \log d})
$$

as required.

Our next statement shows that the multiplicity problem and the height problem are indeed related. Let

$$
s(L, D)=\max r_{1}(f),
$$

where the maximum is taken over all integer polynomials of degree $D$ and of length at most $L$.

Proposition 1. For a given positive integers $L, d$, and an integer irreducible polynomial $P$ of degree $d, d \geq D \geq 2$, we have

$$
s(L, D) \log |P(1)| \leq D \log M(P)+d \log L .
$$

Proof. Assume that $r=s(L, D)>0$, for otherwise the inequality is trivial. Hence, none of the roots of

$$
P(x)=a \prod_{j=1}^{d}\left(x-\alpha_{j}\right)
$$

is a root of $f$, where $r_{1}(f)=r \geq 1$. We have $f(x)=(x-1)^{r} g(x)$ with an integer polynomial $g$. The degree of the latter is equal to $D-r$, thus $\left|a^{D-r} g\left(\alpha_{1}\right) \ldots g\left(\alpha_{d}\right)\right| \geq 1$. On multiplying both sides of this inequality by $|P(1)|^{r}$, we obtain

$$
|P(1)|^{r} \leq|a|^{D}\left\{\prod_{j=1}^{d}\left|\alpha_{j}-1\right|^{r}\right\}\left|g\left(\alpha_{1}\right) \ldots g\left(\alpha_{d}\right)\right|=\left|a^{D} f\left(\alpha_{1}\right) \ldots f\left(\alpha_{d}\right)\right| .
$$


The latter product is less than or equal to $M(P)^{D} L^{d}$ (by the inequality $\left.|f(z)| \leq \max \{1,|z|\}^{D} L\right)$, whence the result.

The best known lower bound for $s(L, D)$ can be used to replace the constant $\pi / 4$ by $1 / \sqrt{2}$ in the above upper bound for $|P(1)|$ provided that $M(P)$ is not too small.

Corollary. Let $0<\nu<2 / 3$. If $P$ is an irreducible integer polynomial of degree $d$, where $d>d(\nu)$, such that $M(P) \geq d^{1 / \nu}$, then

$$
|P(1)| \leq \exp \left(\frac{1+\nu}{\sqrt{2}} \sqrt{d \log d \log M(P)}\right) .
$$

Proof. Fix a small positive constant $\varepsilon$, and let $D$ be sufficiently large. By considering the polynomials with coefficients in $\mathcal{H}$, with $H=\left[D^{1 / \nu} /(D+1)\right]$, and applying Theorem 3 of [2], we get

$$
s(L, D) \geq r_{1}(\mathcal{H}, D)>(1-\varepsilon) \sqrt{8\left(\frac{1}{\nu}-1\right) D} .
$$

Here $L=D^{1 / \nu}$. Taking

$$
d=\left[\frac{\nu D \log M(P)}{\log D}\right]+1 \geq\left[\frac{D \log d}{\log D}\right]+1>\frac{D \log d}{\log D}
$$

we see that $d>D$. On applying the inequality of Proposition 1, we get

$$
\log |P(1)|<(1+2 \varepsilon) \sqrt{\frac{\nu D}{2(1-\nu)}} \log M(P) .
$$

Since $\nu D<(d \log d) / \log M(P)$ and $(1-\nu)^{-1 / 2}<1+\nu$, the statement follows.

The example given in Theorem 2 via Proposition 1 implies the essentially Schur's bound for $s(L, D)$ :

$$
s(L, D) \leq(2+\varepsilon) \sqrt{D \log L} .
$$

A better example would imply stronger bounds.

Suppose $H=\left[D^{\xi}\right]$ with a fixed constant $\xi>0$. From [2], [8] or [23] it follows that

$$
\sqrt{D} \ll r_{1}(\mathcal{H}, D) .
$$

Let $\varphi(d)$ be any function such that $\varphi(d) \rightarrow \infty$ as $d \rightarrow \infty$. Suppose that there exist a positive constant $\gamma$ and a sequence of irreducible integer polynomials $P$ of degree $d$ (where the sequence of the degrees is sufficiently dense) so that $M(P) \geq d^{\varphi(d)}$ and

$$
|P(1)| \geq \exp (\gamma \sqrt{d \log d \log M(P)}) .
$$


Then, taking say $d=[D \log M(P) /(\xi \log D)]$ in Proposition 1, we would get

$$
r_{1}(\mathcal{H}, D) \ll \sqrt{D}
$$

4. Approximation problem. How well can a fixed algebraic number $\beta$ be approximated by algebraic numbers $\alpha$ of small Mahler measure? This question was first investigated by Mignotte [22]. The worst rate of approximation seems to occur in the case when $\beta$ is a root of unity (see also [12]). On replacing $\alpha$ by its power, or by $-\alpha$, if necessary, we see that it suffices to investigate the case $\beta=-1$. The lower bounds for $|\alpha+1|$ were obtained by Mignotte [22], Mignotte and Waldschmidt [25], Bugeaud, Mignotte and Normandin [15], and by the author [16]. For a fixed $\varepsilon>0$ and $d$ sufficiently large the inequality

$$
|\alpha+1|>\exp \left(-\left(\frac{\pi}{4}+\varepsilon\right) \sqrt{d \log d \log M(\alpha)}\right)
$$

is true say in the range $1<M(\alpha)<\exp \left(d^{1-\delta}\right)$ (see [16]).

The existence of algebraic numbers close to 1 or -1 was first shown by Amoroso [3], [4]. The author [17] showed that there are infinitely many algebraic numbers $\alpha$ such that $0<\log M(\alpha) \ll \log d$ (here $d$ is the degree of $\alpha$ ), and

$$
|\alpha+1|<\exp \left(-2.8 \sqrt{\frac{d \log M(\alpha)}{\log d}}\right) .
$$

Proposition 2. For $\alpha \in \overline{\mathbb{Q}}^{*}, \alpha \neq 1$, of degree $d$, we have

$$
|\alpha+1|>\frac{3}{4 d L(\alpha)} \text {. }
$$

Proof. The proposition clearly holds for $d=1$. If $d \geq 2$, then $L(\alpha) \geq 3$. Hence, there is no loss of generality to assume that $|\alpha+1| \leq 1 /(4 d)$. On replacing $\alpha$ by $1 / \alpha$, if necessary, we may assume that $|\alpha| \geq 1$. Therefore

$$
\begin{aligned}
1 & \leq|P(-1)|=|P(-1)-P(\alpha)| \leq|1+\alpha| \max _{1 \leq|z| \leq|\alpha|}\left|P^{\prime}(z)\right| \\
& \leq|1+\alpha| d L(P)\left(1+\frac{1}{4 d}\right)^{d-1}<\frac{4}{3}|1+\alpha| d L(\alpha),
\end{aligned}
$$

and the statement follows.

The above statement is a version of Liouville's inequality. The proof of the first part in the next theorem is essentially the same. The following bounds show the relation between the multiplicity and approximation problems.

Theorem 3. Let $H$ and $D$ be two positive integers. For any root $\alpha$, $\alpha \neq-1$, of a polynomial of degree $D$ with coefficients in $\mathcal{A}$, where $\mathcal{A} \subset \mathcal{H}$, 
we have

$$
|\alpha+1|>\frac{1}{2 H(D+1)}\left(\frac{r_{D}+1}{e D}\right)^{r_{D}+1} .
$$

Here $r_{D}=r_{-1}(\mathcal{A}, D)$.

Furthermore, let $N$ and $s>1$ be two positive integers, even and odd respectively, such that $(N+1)(2 s+1) \leq D+1$, and let $\mathcal{A}$ be a set of complex numbers. Then there is a polynomial of degree at most $D$ with coefficients in $\mathcal{A}$ and a root $\alpha, \alpha \neq-1$, so that

$$
|\alpha+1|<s^{2-r_{N}},
$$

where $r_{N}=r_{-1}(\mathcal{A}, N) \geq 1$.

Proof. The first part is a simplified version of the method used in [12]. Let $f(x)$ be a polynomial of degree $D$ with coefficients in $\mathcal{A}$ so that $r=$ $r_{D}=r_{-1}(\mathcal{A}, D)$. The modulus of the left hand side in the identity

$$
\left.\frac{f(x)}{(x+1)^{r}}\right|_{x=1}=-\left.(1+\alpha) \frac{1}{r !}\left(\frac{f(x)}{x-\alpha}\right)^{(r)}\right|_{x=-1},
$$

where $\alpha, \alpha \neq-1$, is a root of $f$, is bounded below by 1 . There is no loss of generality in assuming that $1 \leq|\alpha| \leq 1+1 /(2(D+1))$. In order to establish the lower bound note that

$$
\begin{aligned}
\left|\frac{1}{r !}\left(\frac{f(x)-f(\alpha)}{x-\alpha}\right)^{(r)}\right|_{x=-1} \mid & \leq \max _{1 \leq|z| \leq|\alpha|}\left|\frac{f^{(r+1)}(z)}{(r+1) !}\right| \\
& \leq \frac{L(f) D^{r+1}|\alpha|^{D-1}}{(r+1) !}<2 H(D+1)\left(\frac{D e}{r+1}\right)^{r+1} .
\end{aligned}
$$

For the second part we use the following statement which is true for any set of complex numbers $\mathcal{A}$.

ObSERVATION. For a given positive integers $N, s$, and a set of complex numbers $\mathcal{A}$, let $f(x)$ be a polynomial of degree $N$ with coefficients in $\mathcal{A}$. Then the coefficients of the polynomial

$$
f(x)+x^{N+1}\left(x^{N s+s}+1\right)\left(x^{s-1}+x^{s-2}+\ldots+1\right) f\left(x^{s}\right)
$$

are all in $\mathcal{A}$.

Let now $f(x)$ be a polynomial of degree $N$ with coefficients in $\mathcal{A}$ so that $r_{-1}(f)=r_{N}=r_{-1}(\mathcal{A}, N)=r$. Set

$$
\begin{aligned}
g(x) & =\frac{f(x)}{(x+1)^{r}}, \\
G(x) & =g(x)+x^{N+1}\left(x^{N s+s}+1\right) \frac{x^{s}-1}{x-1}\left(\frac{x^{s}+1}{x+1}\right)^{r} g\left(x^{s}\right)
\end{aligned}
$$

with $s$ so that $2 N s+2 s+N \leq D$. 
Suppose first that $g^{\prime}(-1) g(-1) \leq 0$. Since $N$ is even and $s>1$ is odd, it follows that

$$
\begin{aligned}
G(-1) & =g(-1) \neq 0, \\
G^{\prime}(-1) & =g^{\prime}(-1)+(-1)^{N+1}(N s+s) s^{r} g(-1) \\
& =g^{\prime}(-1)-(N+1) s^{r+1} g(-1) .
\end{aligned}
$$

Thus, the polynomial $G$ has a root $\alpha, \alpha \neq-1$, such that

$$
\left|\frac{G^{\prime}(-1)}{G(-1)}\right| \leq \frac{\operatorname{deg} G}{|\alpha+1|}=\frac{2 N s+2 s+N-r}{|\alpha+1|}<\frac{3(N+1) s}{|\alpha+1|} .
$$

Here the expression on the left hand side is at least $(N+1) s^{r+1}$, so

$$
|\alpha+1|<\frac{3}{s^{r}} \leq \frac{1}{s^{r-1}} .
$$

Also, $\alpha$ is a root of the polynomial $(x+1)^{r} G(x)$ of degree $2 N s+2 s+N \leq D$ and with coefficients in $\mathcal{A}$ (see Observation).

In the case $g^{\prime}(-1) g(-1)>0$ we take a slightly different polynomial:

$$
G(x)=x^{2 N s+2 s} g(x)+\left(x^{N s+s}+1\right) \frac{x^{s}-1}{x-1}\left(\frac{x^{s}+1}{x+1}\right)^{r} g\left(x^{s}\right) .
$$

This time

$$
\begin{aligned}
G(-1) & =g(-1), \\
G^{\prime}(-1) & =-(2 N s+2 s) g(-1)+g^{\prime}(-1)+(N s+s) s^{r} g(-1) \\
& =g^{\prime}(-1)+(N+1) s\left(s^{r}-2\right) g(-1),
\end{aligned}
$$

and for a root $\alpha, \alpha \neq-1$, of $G$ we have

$$
|\alpha+1|<\frac{3(N+1) s}{(N+1) s\left(s^{r}-2\right)+g^{\prime}(-1) / g(-1)}<\frac{3}{s^{r}-2} \leq \frac{3}{s^{r-1}} \leq \frac{1}{s^{r-2}} .
$$

Again, $\alpha$ is a root of the polynomial

$$
(x+1)^{r} G(x)=x^{2 N s+2 s} f(x)+\left(x^{N s+s}+1\right)\left(x^{s-1}+\ldots+1\right) f\left(x^{s}\right)
$$

of degree $2 N s+2 s+N \leq D$ and with coefficients in $\mathcal{A}$. This completes the proof.

Let $\varepsilon$ be a small positive constant. Taking $s=3$ and applying Theorem 3 to the $\{-1,0,1\}$ polynomials, we obtain

$$
\exp \left(-\left(\frac{\log 3}{\sqrt{7}}+\varepsilon\right) r_{D}\right)<\min |\alpha+1|<\exp \left(-\left(\frac{1}{2}-\varepsilon\right) r_{D} \log D\right),
$$

the minimum being taken over all roots $\alpha, \alpha \neq-1$, of $\{-1,0,1\}$ polynomials of a sufficiently large degree $D$. Recall that here $r_{D}$ is in the range $\sqrt{D / \log D} \ll r_{D}<\frac{21}{13} \sqrt{D}$. 
Write $\{[\sqrt{D / 2}]-1,[\sqrt{D / 2}]-2\}=\{N, s\}$, where $N$ is even and $s$ is odd. Using the bounds

$$
\frac{\log D}{\log 2}-2<r_{D}=r_{-1}(\mathcal{B}, D) \leq \frac{\log D}{\log 2}
$$

(see Theorem 1), from Theorem 3 we now deduce that

$$
\exp \left(-(1+\varepsilon) \frac{(\log D)^{2}}{\log 2}\right)<\min |\alpha+1|<\exp \left(-(1-\varepsilon) \frac{(\log D)^{2}}{4 \log 2}\right) .
$$

Here the minimum is taken over all roots $\alpha, \alpha \neq-1$, of $\{0,1\}$ polynomials of a sufficiently large degree $D$. Borwein and Pinner [12] obtained the constant $4 \log 3=4.394 \ldots$ instead of $4 \log 2=2.772 \ldots$

Finally, taking $N$ and $s$ as above,

$$
\sqrt{D / 2}-3<N, s \leq \sqrt{D / 2}-1
$$

and using Boyd's [13] result (see also [12])

$$
\log D \ll r_{D}=r_{-1}(\mathcal{F}, D) \ll \frac{(\log D)^{2}}{\log \log D},
$$

we have

$$
\exp \left(-(1+\varepsilon) r_{D} \log D\right)<\min |\alpha+1|<\exp \left(-\left(\frac{1}{8}-\varepsilon\right) r_{D} \log D\right)
$$

Here the minimum is taken over all roots $\alpha, \alpha \neq-1$, of $\{-1,1\}$ polynomials of a sufficiently large degree $D$.

Acknowledgements. The author thanks the referee for his careful reading of the manuscript. This research was partially supported by a grant from Lithuanian Foundation of Studies and Science.

\section{References}

[1] F. Amoroso, Sur des polynômes de petites mesures de Mahler, C. R. Acad. Sci. Paris 321 (1995), 11-14.

[2] - Polynomials with prescribed vanishing at roots of unity, Boll. Un. Mat. Ital. B (7) 9 (1995), 1021-1042.

[3] - Algebraic numbers close to 1 and variants of Mahler's measure, J. Number Theory 60 (1996), 80-96.

[4] -, Algebraic numbers close to 1: results and methods, in: Number Theory, V. K. Murthy and M. Waldschmidt (eds.), Contemp. Math. 210, Amer. Math. Soc., Providence, RI, 1998, 305-316.

[5] -, Upper bounds for the resultant and Diophantine applications, in: Number Theory: Diophantine, Computational and Algebraic Aspects, K. Győry, A. Pethő and V. T. Sós (eds.), de Gruyter, Berlin, 1998, 23-36.

[6] P. E. Blanksby and H. L. Montgomery, Algebraic integers near the unit circle, Acta Arith. 18 (1971), 355-369. 
[7] A. Bloch and G. Pólya, On the roots of certain algebraic equations, Proc. London Math. Soc. 33 (1932), 102-114.

[8] E. Bombieri and J. D. Vaaler, On Siegel's lemma, Invent. Math. 73 (1983), 11-32.

[9] -, 一, Polynomials with low height and prescribed vanishing, in: Analytic Number Theory and Diophantine Problems, A. C. Adolphson et al. (eds.), Progr. Math. 70, Birkhäuser, Basel, 1987, 53-73.

[10] P. Borwein, T. Erdélyi and G. Kós, Littlewood-type problems on [0, 1], Bull. London Math. Soc. 79 (1999), 22-46.

[11] P. Borwein and M. G. Mossinghoff, Polynomials with height 1 and prescribed vanishing at 1, Experiment. Math. 9 (2000), 425-433.

[12] P. Borwein and C. Pinner, Polynomials with $\{0,+1,-1\}$ coefficients and a root close to a given point, Canad. J. Math. 49 (1997), 887-915.

[13] D. W. Boyd, On a problem of Byrnes concerning polynomials with restricted coeffcients, Math. Comp. 66 (1997), 1697-1703.

[14] Y. Bugeaud, Algebraic numbers close to 1 in non-archimedean metrics, Ramanujan J. 2 (1998), 449-457.

[15] Y. Bugeaud, M. Mignotte et F. Normandin, Nombres algébriques de petite mesure et formes linéaires en un logarithme, C. R. Acad. Sci. Paris 321 (1995), 517-522.

[16] A. Dubickas, On algebraic numbers of small measure, Liet. Mat. Rink. 35 (1995), 421-431.

[17] -, On algebraic numbers close to 1, Bull. Austral. Math. Soc. 58 (1998), 423-434.

[18] —, Polynomials with high multiplicity at unity and Tarry's problem, Math. Notes 65 (1999), 684-688.

[19] —, On a polynomial with large number of irreducible factors, in: Number Theory in Progress, Vol. 1: Diophantine Problems and Polynomials, K. Győry, H. Iwaniec, J. Urbanowicz (eds.), de Gruyter, Berlin, 1999, 103-110.

[20] - On the order of vanishing at 1 of a polynomial, Liet. Mat. Rink. 39 (1999), 461-468.

[21] - On certain geometric mean of the values of a polynomial, ibid. 40 (2000), 17-27.

[22] M. Mignotte, Approximation des nombres algébriques par des nombres algébriques de grand degré, Ann. Fac. Sci. Toulouse 1 (1979), 165-170.

[23] —, Estimations élémentaires effectives sur les nombres algébriques, Journées Arithmétiques 1980, J. V. Armitage (ed.), London Math. Soc. Lecture Note Ser. 56, Cambridge Univ. Press, 1982, 364-371.

[24] —, An inequality about irreducible factors of integer polynomials, J. Number Theory 30 (1988), 156-166.

[25] M. Mignotte and M. Waldschmidt, On algebraic numbers of small height: linear forms in one logarithm, ibid. 47 (1994), 43-62.

[26] C. Pinner and J. D. Vaaler, The number of irreducible factors of a polynomial. I, Trans. Amer. Math. Soc. 339 (1993), 809-834.

[27] A. Schinzel, On the number of irreducible factors of a polynomial, in: Colloq. Math. Soc. János Bolyai 13, North-Holland, 1976, 305-314.

[28] I. Schur, Untersuchungen über algebraische Gleichungen. I, Bemerkungen zu einem Satz von E. Schmidt, Sitzungsber. Preuss. Acad. Wiss. Phys.-Math. Kl. 7/10 (1933), 403-428.

[29] J. H. Silverman, Lehmer's conjecture and primes of small norm, Brown University, preprint, 1994.

[30] -, Exceptional units and numbers of small Mahler measure, Experiment. Math. 4 (1995), 69-83. 
[31] J. H. Silverman, Small Salem numbers, exceptional units, and Lehmer's conjecture, Rocky Mountain J. Math. 26 (1996), 1099-1114.

[32] D. Zagier, Algebraic numbers close to both 0 and 1, Math. Comp. 61 (1993), 485-491.

Department of Mathematics and Informatics

Vilnius University

Naugarduko 24

2600 Vilnius, Lithuania

E-mail: arturas.dubickas@maf.vu.lt

Received on 16.3.2000

and in revised form on 3.8.2000 